\title{
Psoralidin induces autophagy through ROS generation which inhibits the proliferation of human lung cancer A549 cells
}

Psoralidin (PSO), a natural furanocoumarin, is isolated from Psoralea corylifolia L.

possessing anti-cancer properties. However, the mechanisms of its effects remain unclear. Herein, we investigated its anti-proliferative effect and potential approaches of action on human lung cancer A549 cells. Cell proliferation and death were measured by MTT and LDH assay respectively. Apoptosis was detected with Hoechst 33342 staining by fluorescence microscopy, Annexin V-FITC by cytometry and Western blot analysis for apoptosis-related proteins. The autophagy was evaluated using MDC staining, immunofluorescence assay and Western blot analyses for LC3-I and LC3-II. In addition, the reactive oxygen species (ROS) generation was measured by DCFH2-DA with flow cytometry. PSO dramatically decreased the cell viabilities in dose- and time- dependent manner. However, no significant change was observed between the control group and the PSO-treated groups in Hoechst 33342 and Annexin V-FITC staining. The expression of apoptosis-related proteins was not altered significantly as well. While the MDC-fluorescence intensity and the expression ratio of LC3II/LC3-I was remarkably increased after PSO treatment. Autophagy inhibitor 3-MA blocked the production of LC3-II and reduced the cytotoxicity in response to PSO. Furthermore, PSO increased intracellular ROS level which was correlated to the elevation of LC3-II. ROS scavenger $\mathrm{N}$-acetyl cysteine pretreatment not only decreased the ROS level, reduced the expression of LC3-II but also reversed PSO induced cytotoxicity. PSO inhibited the proliferation of A549 cells through autophagy but not apoptosis, which was mediated by inducing ROS production. 
1 Psoralidin induces autophagy through ROS generation which inhibits the proliferation of

2

3

4

5

6 Running title: Psoralidin induces autophagy

$7 \quad *$ Send correspondence to: Dr. Xiuping Chen

8 Address: Institute of Chinese Medical Sciences, University of Macau, Av. Padre Tomas Pereira S.J.,

9 Taipa, Macau, China

10 xpchen@umac.mo

11 Tel: $+853-88224679$

12 tCo-first author.

14 Psoralidin (PSO), a natural furanocoumarin, is isolated from Psoralea corylifolia L. possessing 15 anti-cancer properties. However, the mechanisms of its effects remain unclear. Herein, we 
16 investigated its anti-proliferative effect and potential approaches of action on human lung cancer

17 A549 cells. Cell proliferation and death were measured by MTT and LDH assay respectively.

18 Apoptosis was detected with Hoechst 33342 staining by fluorescence microscopy, Annexin V-

19 FITC by cytometry and Western blot analysis for apoptosis-related proteins. The autophagy was

20 evaluated using MDC staining, immunofluorescence assay and Western blot analyses for LC3-I

21 and LC3-II. In addition, the reactive oxygen species (ROS) generation was measured by $\mathrm{DCFH}_{2}$ -

22 DA with flow cytometry. PSO dramatically decreased the cell viabilities in dose- and time-

23 dependent manner. However, no significant change was observed between the control group and

24 the PSO-treated groups in Hoechst 33342 and Annexin V-FITC staining. The expression of 25 apoptosis-related proteins was not altered significantly as well. While the MDC-fluorescence 26 intensity and the expression ratio of LC3-II/LC3-I was remarkably increased after PSO treatment. 27 Autophagy inhibitor 3-MA blocked the production of LC3-II and reduced the cytotoxicity in 28 response to PSO. Furthermore, PSO increased intracellular ROS level which was correlated to the 29 elevation of LC3-II. ROS scavenger N-acetyl cysteine pretreatment not only decreased the ROS 30 level, reduced the expression of LC3-II but also reversed PSO induced cytotoxicity. PSO 31 inhibited the proliferation of A549 cells through autophagy but not apoptosis, which was 32 mediated by inducing ROS production.

33 Keywords: Psoralidin; ROS; Autophagy; Cancer

\section{INTRODUCTION}

35 Psoralea corylifolia Leguminosae (L.), an herb widely distributed in China and Southeastern 36 Asian countries, has been used as a multi-purpose medicinal plant (Zhao et al., 2005). The fully 37 mature dried fruit of this plant is well-known as traditional Chinese medicine called "Buguzhi", 38 and is used to treat a wide range of diseases. Previous screening studies have reported that some 39 extracts and active fractions of $P$. corylifolia $L$. exhibited cytotoxicity and inhibition of chemical 
41 major chemical constituents in P. corylifolia L. include coumarins, flavonoids and meroterpene 42 phenols ( 43 bioactive components. It is reported that PSO shows cytotoxic effects against some cultured 44 human cancer cell lines (Bronikowska et al., 2012; Kumar et al., 2010; Mar et al., 2001; $\underline{\text { Pahari }}$ 45 46 47

\section{MATERIALS AND METHODS}

\section{Reagents and cell culture}

55 Psoralidin (>98\%) was purchased from Chengdu Preferred Biotech Co. Ltd (Chengdu $\square$ China).

56 Dimethyl sulfoxide (DMSO), MTT, Hoechst 33342, monodansylcadaverine (MDC), propidium 57 iodide (PI), 3-methyladenine (3-MA), Ac-DEVD-CHO, z-VAD-FMK, DAPI, CM-H ${ }_{2}$ DCF-DA, 58 N-acetyl cysteine (NAC), Annexin V-FITC were from Sigma Aldrich (St. Louis, MO, USA). 59 Cytotoxicity Detection Kit (lactate dehydrogenase, LDH) was obtained from Roche Diagnostics 60 (Mannheim, Germany). Antibodies against LC3, Bcl-2, BAX, PARP, Caspase-3, Caspase-9 and 61 GAPDH were purchased from Cell Signaling Technology (Beverly, MA, USA).

62 The human lung cancer cell line A549, obtained from American Type Culture Collection 63 (ATCC, USA), was cultured in RPMI 1640 (Gibco) supplemented with 10\% (v/v) fetal bovine 
64 serum at $37^{\circ} \mathrm{C}$ in a humidified atmosphere of $5 \% \mathrm{CO}_{2}$.

\section{MTT assay and LDH assay}

66 Cells in the exponential growth phase were seeded in 96-well culture plates (5000 cells per well),

67 treated with various concentrations $(1.25,2.5,5,10,20,30$ and $40 \mu \mathrm{M})$ of PSO for indicated 68 time. After incubation, $20 \mu \mathrm{MTT}$ solutions $(5 \mathrm{mg} / \mathrm{ml})$ was added to each well and incubated for

69 further $4 \mathrm{~h}$. Then the supernatant was removed and the resulting crystals were dissolved in

70 DMSO. The absorbance of each well was measured using a microplate reader (PerkinElmer,

71 USA) at $570 \mathrm{~nm}$. The cell viability was calculated by the formula: cell viability $(\%)=($ average

72 absorbance of treated groups/average absorbance of control group) $\times 100 \%$. A commercial

73 cytotoxicity detection kit was used to evaluate the LDH release from cells after treatment with

74 different concentrations of PSO according to the manufacturer's protocol.

\section{Cell cycle analysis}

76 After treated with PSO, cells were harvested and washed with cold phosphate buffer saline 77 (PBS), and were fixed with $70 \%$ ethanol overnight at $-20^{\circ} \mathrm{C}$. The fixed cells were then washed 78 twice with cold PBS, and the supernatant was removed. Cells were stained with PI staining 79 solution $(10 \mu \mathrm{g} / \mathrm{ml} \mathrm{RNase} \mathrm{A}$ and $50 \mu \mathrm{g} / \mathrm{ml} \mathrm{PI})$ at $37^{\circ} \mathrm{C}$ for $30 \mathrm{~min}$ in dark. The cell cycle 80 distribution was analyzed using a flow cytometry provided with the Cell-Quest software (Becton 81 Dickinson, USA).

\section{Apoptosis detection}

83 Hoechst 33342 staining and Annexin V-FITC staining were performed to detect apoptosis. For 84 Hoechst 33342 staining, A549 cells were washed with PBS and stained with Hoechst 33342 (1 $85 \mu \mathrm{g} / \mathrm{ml}$ in PBS) at room temperature for $20 \mathrm{~min}$, the fluorescence was observed by a fluorescence 86 inverted microscopy. For Annexin V-FITC staining, the treated cells were collected, washed and 87 then stained with Annexin V-FITC at room temperature for $15 \mathrm{~min}$, the percentage of apoptotic 88 cells were analyzed by flow cytometry. 
MDC staining and immunofluorescence

90 The autophagic activity was evaluated using MDC staining and immunofluorescence for LC3-II

91 by fluorescence microscopy as described previously (Zhang et al., 2013). In brief, the treated

92 cells were incubated with $0.05 \mathrm{mM} \mathrm{MDC}$ for $15 \mathrm{~min}$ in the dark, then washed with PBS twice

93 and immediately analyzed by a fluorescence inverted microscopy. For immunofluorescence, the

94 treated cells were fixed with $4 \%$ paraformaldehyde and blocked with $2 \%$ BSA for 30 min,

95 incubated with primary antibody against LC3-II at $4^{\circ} \mathrm{C}$ overnight, then washed with PBS twice

96 and incubated with fluo-conjugated secondary antibody at room temperature for another $1 \mathrm{~h}$. The

97 nuclei were stained with DAPI and the stained cells were observed under a fluorescence inverted

98 microscope using filter set for FITC and DAPI.

99 Western blot analysis

100 After treated with PSO, cells were washed with cold PBS and lysed with RIPA lysis buffer (Santa

101 Cruz, USA) to extract the total proteins. The concentrations of the total proteins were determined 102 by Pierce BCA protein assay kit (Thermo Scientific, USA). Equivalent amounts of total proteins 103 from each sample were separated by SDS-PAGE, and then transferred onto a PVDF membrane. 104 After blocking with PBS containing 0.1\% Tween-20 and 5\% nonfat milk for $1 \mathrm{~h}$, the transferred 105 membrane was incubated with a specific primary antibody at $4^{\circ} \mathrm{C}$ overnight, followed by 106 incubation with the corresponding secondary antibody. Washing the membrane and specific 107 protein bands were visualized using an ECL Advanced Western Blot detection Kit, the 108 densitometric analysis of bands was performed using the Quantity-One Software.

109 Determination intracellular ROS production

110 Intracellular ROS production was measured by flow cytometry analysis using $\mathrm{CM}-\mathrm{H}_{2} \mathrm{DCF}-\mathrm{DA}$ as

111 fluorescence probe. After PSO treatment, cells were washed and incubated with $\mathrm{DCFH}_{2}-\mathrm{DA}$ $112(5 \mu \mathrm{M})$ for $30 \mathrm{~min}$ at $37^{\circ} \mathrm{C}$ in the dark. The stained cells were analyzed by flow cytometry.

\section{Statistical analysis}

PeerJ reviewing PDF | (v2014:05:2153:1:4:NEW 30 Jul 2014) 
114 Data are expressed as the means \pm SD from at least three independent experiments. The

115 differences between groups were analyzed by one-way ANOVA with Tukey's posthoc tests,

116 significance of difference was indicated as $* P<0.05$ or $* * P<0.01$.

\section{RESULTS}

\section{The cytotoxicity of PSO in A549 cells}

119 The effects of PSO in A549 cells were detected using MTT assay. As shown in Fig. 1B, the 120 inhibition of PSO in A549 cell proliferation was exhibited both in time- and concentration121 dependent manners. The calculated $\mathrm{IC}_{50}$ after 24,48 and $72 \mathrm{~h}$ treatment were $19.2,15.4$ and 11.8 $122 \mu \mathrm{M}$ respectively. Furthermore, after treatment with varies concentrations of PSO for $24 \mathrm{~h}$, the 123 LDH release from A549 cells was increased in a concentration-dependent manner (Fig. 1C).

\section{PSO induced cell cycle arrest at G1 phase}

125 To measure the underlying mechanism responsible for the anti-proliferation effect of PSO in 126 A549 cells, cell cycle distribution was detected by flow cytometry analysis of DNA content using 127 PI staining. As shown in Fig. 2, PSO in concentrations of 10 and $20 \mu \mathrm{M}$ induced an increase in 128 the percentage of cells in G1 phase. Compared with the control group, $20 \mu \mathrm{M}$ PSO treatment 129 groups showed significant increase $(P<0.05)$ in the proportion of G1 phase cells (Fig. 2B).

\section{PSO showed little effect on apoptosis in A549 cells}

131 Hoechst 33342 staining and flow cytometry assay using Annexin V-FITC staining were

132 performed to determine whether apoptosis was induced by PSO. As shown in Fig. 3A, both the 133 untreated and treated cells had regular and round-shaped nuclei and the characteristic 134 morphological changes of apoptosis were not observed. Flow cytometry analysis using Annexin 135 V-FITC staining showed that treatment A549 cells with different concentrations of PSO did not 136 alter the percentage of Annexin V-FITC positive cells compared with control group (Fig. 3B). 137 Furthermore, the expressions of some apoptosis-related proteins were analyzed by Western blot. 138 After the treatment with PSO for $24 \mathrm{~h}$, the expression of apoptosis-related proteins Bcl-2, BAX, 
139 Caspase-3, Caspase-9 and PARP did not alter significantly (Fig. 3C). These results suggest that

140 PSO maybe not induce apoptosis in A549 cells.

141 In addition, the pancaspase inhibitor Z-VAD-FMK and caspse-3 inhibitor AC-DEVD-CHO

142 were used to confirm the effect of PSO in A549 cells. As shown in Fig. 3D, Z-VAD-FMK and

143 AC-DEVD-CHO failed to protect A549 cells from death caused by PSO. These results

144 collectively indicate that the cell death induced by PSO is in a caspase-independent non-apoptotic

145 manner.

146 PSO induced autophagic cell death in A549 cells

147 To test the autophagic activity induced by PSO, MDC staining was performed firstly. As shown

148 in Fig. 4A, the control cells showed faint fluorescence, while cells treated with PSO accumulated

149 MDC into granular structures of high fluorescence intensity. The immunofluorescence for LC3-II

150 was executed to further examine the formation of autophagic vesicles. Compared with the

151 untreated cells, A549 cells treated with PSO exhibited increases in both the number and size of

152 LC3-II-postive puncta (Fig. 4B). Western blot analysis for LC3 showed a remarkable increase of

153 LC3-II in response to PSO treatment both in concentration- and time-dependent manner (Fig. 4C

154 and D). Furthermore, this increase of LC3-II could be blocked in the presence of autophagy

155 inhibitor 3-MA (Fig. 4E). In addition, pretreatment with 3-MA significantly prevented cell death

156 induced by PSO (Fig. 4 F). Taken together, these results suggest that PSO induces autophagic cell

157 death instead of apoptosis in A549 cells.

158 PSO induced ROS generation and NAC reversed PSO-induced autophagy and cell death

159 As shown in Fig. 5A and B, PSO induced ROS generation in a concentration-dependent manner

160 in A549 cells. NAC pretreatment efficiently attenuated PSO induced elevation of ROS levels.

161 Meanwhile, pretreatment with NAC prevented the increase of LC3-II and cell death in response

162 to PSO (Fig. 5C and D). These results suggested that the production of ROS plays a key role in

163 the PSO-induced autophagy and subsequent cell death. 


\section{DISCUSSION}

165 PSO is natural product isolated from Psoralea corylifolia L., which is an important medical herb

166 prescribed in traditional Chinese medicine. Previous reports demonstrated that PSO showed

167 significant antibacterial (Khatune et al., 2004), antidepressant-like properties (Yi et al., 2008),

168 antioxidant ( $\underline{\text { Xiao et al., 2010) }}$ activities. It also showed inhibitory effect on protein tyrosine

169 phosphatase 1B in vitro ( $\underline{\text { Kim et al., 2005) }}$, induction of quinone reductase activity ( Lee et al.

170 2009), and inhibitory effect on LPS-induced iNOS expression (Chiou et al.,2011). Furthermore, it

171 was found that PSO could act as an ER agonist (Liu et al., 2014) and was a dual inhibitor of

172 COX-2 and 5-LOX (Yang et al., 2011). Herein, the anti-proliferative effect of PSO was

173 investigated.

174 Previous studies showed that PSO inhibited the proliferation of SNU-1 and SNU-16 gastric 175 carcinoma cell lines (Yang et al., 1996), HT-29 colon and MCF-7 breast human cancer cell lines

176 (Mar et al., 2001). In present study, we showed that PSO inhibited proliferation on human lung

177 cancer $\mathrm{A} 549$ cells with $\mathrm{IC}_{50}$ at the range of 10 to $20 \mu \mathrm{M}$, which suggested PSO possesses a

178 potential cytotoxicity to cancer cells. This was further confirmed by PSO induced LDH release in

179 the culture medium. The effect of PSO on cell showed that PSO induced an increase in the 180 percentage of cells in G1 phase, suggesting PSO could cause the cell cycle arrest.

181 Apoptosis, a physiological process of programmed cell death, is one of the major types of

182 cell death caused by most chemotherapeutics. It has been reported that PSO enhanced TRAIL-

183 induced apoptosis in HeLa cells $(\underline{\text { Bronikowska et al., 2012) }})$ and prostate cancer cells ( $\underline{\text { Szliszka et }}$

184 al., 2011). In present study, the effect of PSO on apoptosis in A549 cells was detected using

185 Hoechst 33342 staining by fluorescence microscopy and Annexin V-FITC staining by flow

186 cytometry. In addition, the expressions of apoptosis-related proteins such as Bcl-2, Bax, Caspase-

187 3, Caspase-9 and PARP were examined by Western blot. There was no significant nuclear 
188 changes in Hoechst 33342 staining were observed. Furthermore, no significant differences in the

189 percentage of Annexin V-FITC positive cells and apoptosis-related proteins expression between

190 the control groups and PSO treated groups. In addition, the pancaspase inhibitor Z-VAD-FMK

191 and caspse-3 inhibitor AC-DEVD-CHO failed to protect A549 cells from PSO induced death.

192 Taken together, these results indicated that apoptosis might play a minor role in PSO induce cell 193 death in A549 cells.

194 Autophagy, a cellular process responsible for the degradation of cytoplasmic components 195 through an autophagosomal-lysosomal pathway, has been implicated to play a key role in cancer

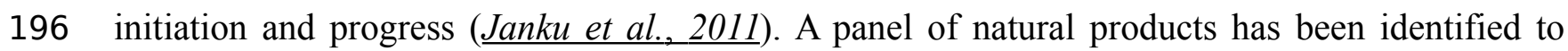
197 induce both apoptotic and autophagic cell death. PSO showed cytotoxicity to A549 cells but did 198 not induce apoptosis. Therefore, we detected whether PSO induced autophagic cell death in A549 199 cells. MDC is an acidotropic dye that labels late stage autophagosomes or autophagic vesicles. 200 During autophagosome formation, LC3-I is incorporated into autophagosome membranes 201 mediated by Atg3 and Atg7, which results in the conversion of cytosolic LC3-I into membrane202 bound form LC3-II (Tanida et al., 2004). Thus, the both the MDC staining and the expression 203 ratio of LC3-I to LC3-II provides simple indicators for autophagy activity and has been widely 204 used to monitor the autophagic activities (Klionsky et al., 2012). Present results showed that PSO 205 induced MDC staining, punctate staining dots for LC3-II $\square$ and increased protein expression ratio 206 of LC3-II/LC3-I suggesting that PSO caused autophagy in A549 cells. Furthermore, pretreatment 207 with 3-MA, an autophagy inhibitor, reversed PSO induced significantly protein expression ratio 208 of LC3-II/LC3-I and cell death induced by PSO. Taken together, these results suggested that PSO 209 induced autophagic cell death instead of apoptosis in A549 cells. To the best of our knowledge, 210 this is first report on the effect of PSO on autophagy.

211 Accumulated evidence suggested that ROS, a class of important multifaceted signaling 212 molecules implicated in a variety of cellular programs including autophagy (Azad et al., 2009; 
213 Dewaele et al., 2010; Scherz-Shouval and Elazar, 2011). Though PSO showed anti-oxidant

214 activities in scavenging DPPH and ABTS free radicals in ex vivo models (Wang et al., 2013; $\underline{\text { Xiao }}$

215 et al.,2010), it greatly induced ROS generation that resulted in the growth inhibition and promote

216 epithelial-mesenchymal transition in prostate cancer cells (Das et al., 2013). We also found that

217 PSO significantly induced DCF fluorescence in A549 cells suggesting the increase of

218 intracellular ROS generation. NAC, a ROS scavenger, pretreatment could significantly reverse

219 PSO induced autophagic biomarkers and cell death suggested that ROS mediated PSO-induced

220 autophagy and subsequent cell death.

221 In summary, as shown in Fig 6, our results show that PSO is a potential cytotoxic natural

222 compound. Instead of apoptosis, it induces ROS-triggered autophagy which inhibits the growth

223 of human lung cancer A549 cells.

\section{Conflicts of interest}

225 We declare that none of the authors has any conflict of interest related to the present work.

\section{REFERENCE}

227 Azad MB, Chen Y, Gibson SB, 2009. Regulation of autophagy by reactive oxygen species (ROS): implications for 228 cancer progression and treatment. Antioxidants \& redox signaling 11, 777-790.

229 Bronikowska J, Szliszka E, Jaworska D, Czuba ZP, Krol W, 2012. The coumarin psoralidin enhances anticancer 230 effect of tumor necrosis factor-related apoptosis-inducing ligand (TRAIL). Molecules 17, 6449-6464.

Chiou WF, Don MJ, Liao JF, Wei BL, 2011. Psoralidin inhibits LPS-induced iNOS expression via repressing Sykmediated activation of PI3K-IKK-IkappaB signaling pathways. Eur J Pharmacol 650, 102-109.

Das TP, Suman S, Damodaran C, 2013. Reactive oxygen species generation inhibits epithelial-mesenchymal transition and promotes growth arrest in prostate cancer cells. Molecular carcinogenesis.

Dewaele M, Maes H, Agostinis P, 2010. ROS-mediated mechanisms of autophagy stimulation and their relevance in cancer therapy. Autophagy 6, 838-854.

Janku F, McConkey DJ, Hong DS, Kurzrock R, 2011. Autophagy as a target for anticancer therapy. Nature reviews. Clinical oncology 8, 528-539.

Khatune NA, Islam ME, Haque ME, Khondkar P, Rahman MM, 2004. Antibacterial compounds from the seeds of Psoralea corylifolia. Fitoterapia 75, 228-230.

Kim YC, Oh H, Kim BS, Kang TH, Ko EK, Han YM, Kim BY, Ahn JS, 2005. In vitro protein tyrosine phosphatase 1B inhibitory phenols from the seeds of Psoralea corylifolia. Planta Med 71, 87-89.

Klionsky DJ, Abdalla FC, Abeliovich H, Abraham RT, Acevedo-Arozena A, Adeli K, Agholme L, Agnello M, Agostinis P, Aguirre-Ghiso JA, et al., 2012. Guidelines for the use and interpretation of assays for monitoring autophagy. Autophagy 8, 445-544.

Kumar R, Srinivasan S, Pahari P, Rohr J, Damodaran C, 2010. Activating stress-activated protein kinasemediated cell death and inhibiting epidermal growth factor receptor signaling: a promising therapeutic strategy for 
prostate cancer. Molecular cancer therapeutics 9, 2488-2496.

249 Latha PG, Evans DA, Panikkar KR, Jayavardhanan KK, 2000. Immunomodulatory and antitumour properties of 250 Psoralea corylifolia seeds. Fitoterapia 71, 223-231.

251 Latha PG, Panikkar KR, 1999. Inhibition of chemical carcinogenesis by Psoralea corylifolia seeds. Journal of 252 ethnopharmacology 68, 295-298.

253 Lee SJ, Nam KW, Mar W, 2009. Induction of quinone reductase activity by psoralidin isolated from Psoralea 254 corylifolia in mouse hepa 1c1c7 cells. Arch Pharm Res 32, 1061-1065.

255 Liu X, Nam JW, Song YS, Viswanath AN, Pae AN, Kil YS, Kim HD, Park JH, Seo EK, Chang M, 2014. 256 Psoralidin, a coumestan analogue, as a novel potent estrogen receptor signaling molecule isolated from Psoralea corylifolia. Bioorganic \& medicinal chemistry letters.

Mar W, Je KH, Seo EK, 2001. Cytotoxic constituents of Psoralea corylifolia. Arch Pharm Res 24, 211-213. Pahari P, Rohr J, 2009. Total synthesis of psoralidin, an anticancer natural product. The Journal of organic chemistry 74, 2750-2754.

Scherz-Shouval R, Elazar Z, 2011. Regulation of autophagy by ROS: physiology and pathology. Trends in biochemical sciences $\mathbf{3 6}, 30-38$.

Song P, Yang XZ, Yuan JQ, 2013. Cytotoxic constituents from Psoralea corylifolia. Journal of Asian natural products research $\mathbf{1 5}, 624-630$.

Srinivasan S, Kumar R, Koduru S, Chandramouli A, Damodaran C, 2010. Inhibiting TNF-mediated signaling: a novel therapeutic paradigm for androgen independent prostate cancer. Apoptosis 15, 153-161.

Suman S, Das TP, Damodaran C, 2013. Silencing NOTCH signaling causes growth arrest in both breast cancer stem cells and breast cancer cells. British journal of cancer 109, 2587-2596.

Szliszka E, Czuba ZP, Sedek L, Paradysz A, Krol W, 2011. Enhanced TRAIL-mediated apoptosis in prostate cancer cells by the bioactive compounds neobavaisoflavone and psoralidin isolated from Psoralea corylifolia. Pharmacol Rep 63, 139-148.

Tanida I, Ueno T, Kominami E, 2004. LC3 conjugation system in mammalian autophagy. The international journal of biochemistry \& cell biology 36, 2503-2518.

Wang TX, Yin ZH, Zhang W, Peng T, Kang WY, 2013. [Chemical constituents from Psoralea corylifolia and their antioxidant alpha-glucosidase inhibitory and antimicrobial activities]. Zhongguo Zhong Yao Za Zhi 38, 2328-2333.

Whelan LC, Ryan MF, 2003. Ethanolic extracts of Euphorbia and other ethnobotanical species as inhibitors of human tumour cell growth. Phytomedicine : international journal of phytotherapy and phytopharmacology 10, 5358.

Xiao G, Li G, Chen L, Zhang Z, Yin JJ, Wu T, Cheng Z, Wei X, Wang Z, 2010. Isolation of antioxidants from Psoralea corylifolia fruits using high-speed counter-current chromatography guided by thin layer chromatographyantioxidant autographic assay. Journal of chromatography. A 1217, 5470-5476.

Yang HJ, Youn H, Seong KM, Yun YJ, Kim W, Kim YH, Lee JY, Kim CS, Jin YW, Youn B, 2011. Psoralidin, a dual inhibitor of COX-2 and 5-LOX, regulates ionizing radiation (IR)-induced pulmonary inflammation. Biochemical pharmacology 82, 524-534.

Yang YM, Hyun JW, Sung MS, Chung HS, Kim BK, Paik WH, Kang SS, Park JG, 1996. The cytotoxicity of psoralidin from Psoralea corylifolia. Planta Med 62, 353-354.

Yi LT, Li YC, Pan Y, Li JM, Xu Q, Mo SF, Qiao CF, Jiang FX, Xu HX, Lu XB, et al., 2008. Antidepressant-like effects of psoralidin isolated from the seeds of Psoralea Corylifolia in the forced swimming test in mice. Prog Neuropsychopharmacol Biol Psychiatry 32, 510-519.

Zhang X, Wei H, Liu Z, Yuan Q, Wei A, Shi D, Yang X, Ruan J, 2013. A novel protoapigenone analog RY10-4 induces breast cancer MCF-7 cell death through autophagy via the Akt/mTOR pathway. Toxicology and applied pharmacology 270, 122-128.

Zhao L, Huang C, Shan Z, Xiang B, Mei L, 2005. Fingerprint analysis of Psoralea corylifolia L. by HPLC and LCMS. Journal of chromatography. B, Analytical technologies in the biomedical and life sciences 821, 67-74. 


\section{Figures and legends:}

296 Fig. 1. The cytotoxicity of psoralidin against human lung cancer A549 cells. (A) Chemical 297 structure of psoralidin. (B) Cells were treated with series concentrations of psoralidin for 24, 48, 298 or $72 \mathrm{~h}$. The cell viability was measured by MTT assay, and the data were presented as means \pm 299 SD from three independent experiments. (C) Cells were treated with 5, 10, 20 and $30 \mu \mathrm{M}$ 300 psoralidin for $24 \mathrm{~h}$, the LDH assay were performed. ${ }^{*} P<0.05$ vs. Con and ${ }^{* *} P<0.01 v s$. Con. 301 Con, control

302 Fig. 2. Effect of psoralidin in cell cycle distribution of A549 cells. (A) Cells were treated with 5, 30310 and $20 \mu \mathrm{M}$ psoralidin for $24 \mathrm{~h}$, and then stained with propidium iodide. The DNA content was 304 measured by flow cytometry. (B) The cell cycle distributions were analyzed and presented as 305 mean $\pm \mathrm{SD}$ of three independent experiments. $* P<0.05$ vs. Con. Con, control

306 Fig. 3. Psoralidin showed little effect on apoptosis in A549 cells. (A) Fluorescent staining of 307 nuclei in A549 cells by Hoechst 33342. (B) Flow cytometry assay to detect apoptosis in A549 308 cells using Annexin V staining. There is no significant difference among control group and 309 psoralidin treated groups. NS, no significant vs. Con. (C) The expression of apoptosis related 310 proteins were analyzed by Western blot in A549 cells receiving psoralidin treatment. (D) The cell

311 viability was measured by MTT assay after treatment with psoralidin in the absence or presence 312 of Z-VAD-FMK or Ac-DEVD-CHO. $* * P<0.01$ vs. Con; NS, no significant vs. psoralidin alone313 treated group. Con, control

314 Fig. 4. Psoralidin induced autophagic cell death in A549 cells. (A) Cells were stained with MDC 
315 after psoralidin treatment. (B) Immunofluerescence analysis of endogenous LC3 in psoralidin316 treated A549 cells. (C) Cells were treated with indicated concentrations of psoralidin for $24 \mathrm{~h}$. 317 The expression of LC3 was determined by Western blot. (D) Cells were treated with $20 \mu \mathrm{M}$ 318 psoralidin for 6, 12, 24 or 48 h, expression of LC3 was determined by Western blot. (E) Cells 319 were treated with psoralidin, 3-MA or a combination of both, expression of LC3 was analyzed by 320 Western blot. (F) The cell viability was measured by MTT assay after treatment with psoralidin in 321 the absence or presence of 3-MA. ${ }^{* *} P<0.01 v s$. Con, $\# P<0.05$ vs. psoralidin alone-treated 322 group. Con, control

323 Fig. 5. Psoralidin induced ROS generation and NAC reversed psoralidin-induced autophagy and 324 cell death. (A) Cells were treated with indicated concentrations of psoralidin, and the intracellular 325 ROS generation was detected as described in Materials and methods. ${ }^{*} P<0.05$ vs. Con and ${ }^{*} P$ $326<0.01$ vs. Con. (B) Cells were treated with $20 \mu \mathrm{M}$ psoralidin in the absence or presence of NAC. 327 The intracellular ROS generation was detected, and data represent the means \pm SD from three 328 independent experiments. ${ }^{* *} P<0.01$ vs. Con, ${ }^{\#} P<0.05$ vs. psoralidin alone-treated group. (C) 329 Cells were treated with $20 \mu \mathrm{M}$ psoralidin in the absence or presence of NAC. The expression of 330 LC3 was analyzed by Western blot. (D) The cell viability was measured by MTT assay after 331 psoralidin with treatment in the absence or presence of NAC. ${ }^{* *} P<0.01$ vs. Con, ${ }^{\sharp} P<0.05$ vs. 332 psoralidin alone-treated group. Con, control

333 Fig. 6. Schematic diagram illustrates the underlying mechanism of psoralidin-induced cell death 334 in A549 cells. 


\section{Figure 1}

Fig. 1. The cytotoxicity of psoralidin against human lung cancer A549 cells.

(A) Chemical structure of psoralidin. (B) Cells were treated with series concentrations of psoralidin for 24,48 , or $72 \mathrm{~h}$. The cell viability was measured by MTT assay, and the data were presented as means \pm SD from three independent experiments. (C) Cells were treated with 5, 10, 20 and $30 \mu \mathrm{M}$ psoralidin for $24 \mathrm{~h}$, the LDH assay were performed. ${ }^{*} P<0.05$ vs. Con and ${ }^{\star *} P<0.01$ vs. Con. Con, control $\langle$ ?xml:namespace prefix = o ns = "urn:schemas-microsoft-com:office:office" $/\rangle$
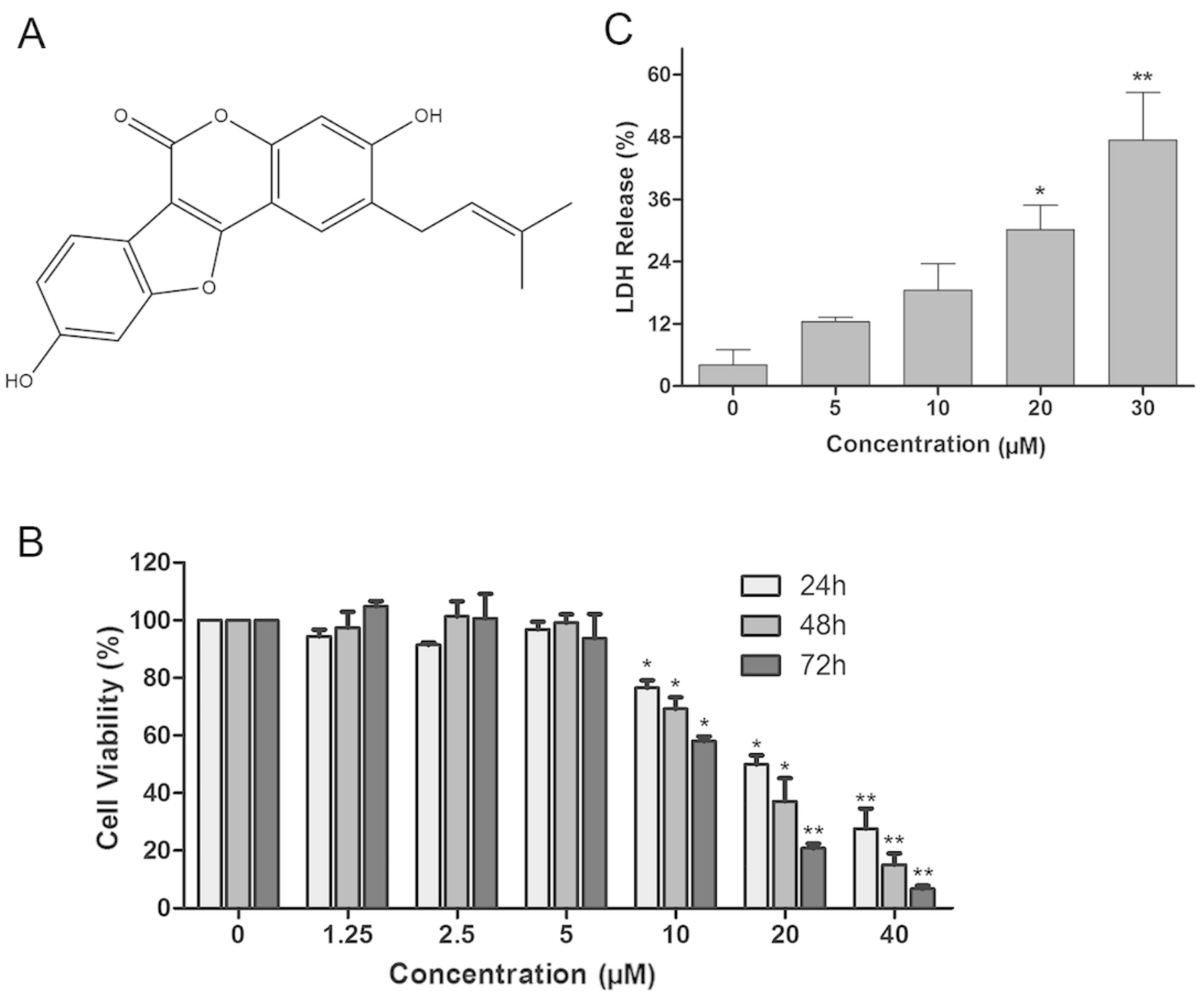


\section{Figure 2}

Fig. 2. Effect of psoralidin in cell cycle distribution of A549 cells.

(A) Cells were treated with 5, 10 and $20 \mu \mathrm{M}$ psoralidin for $24 \mathrm{~h}$, and then stained with propidium iodide. The DNA content was measured by flow cytometry. (B) The cell cycle distributions were analyzed and presented as mean \pm SD of three independent experiments. ${ }^{*} P<0.05$ vs. Con. Con, control $<$ ?xml:namespace prefix $=0 \mathrm{~ns}=$ "urn:schemas-microsoft-com:office:office" $/\rangle$
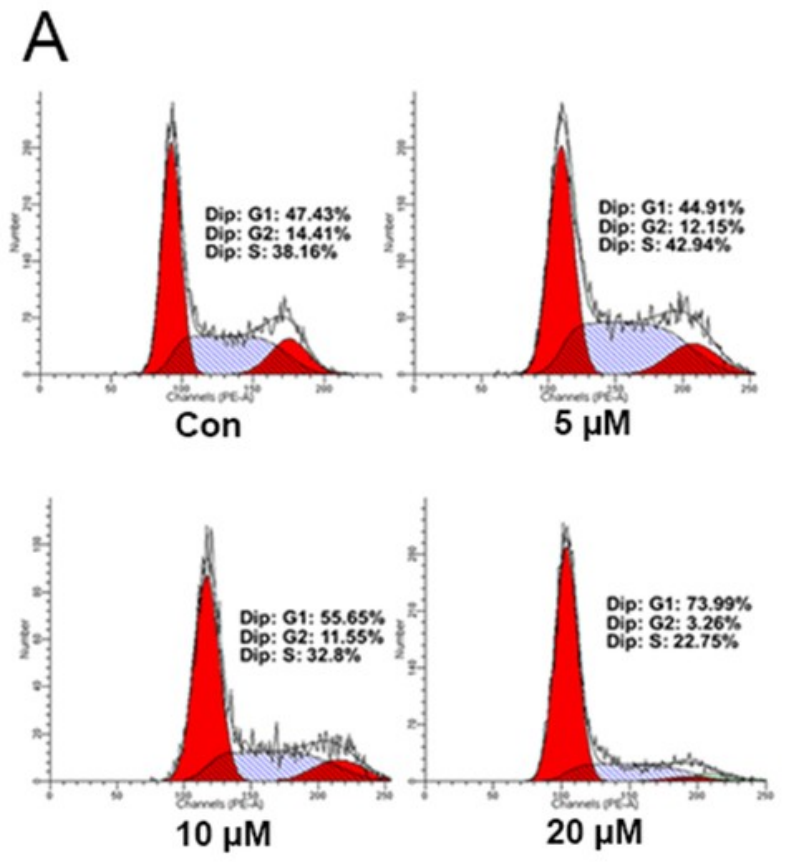

B

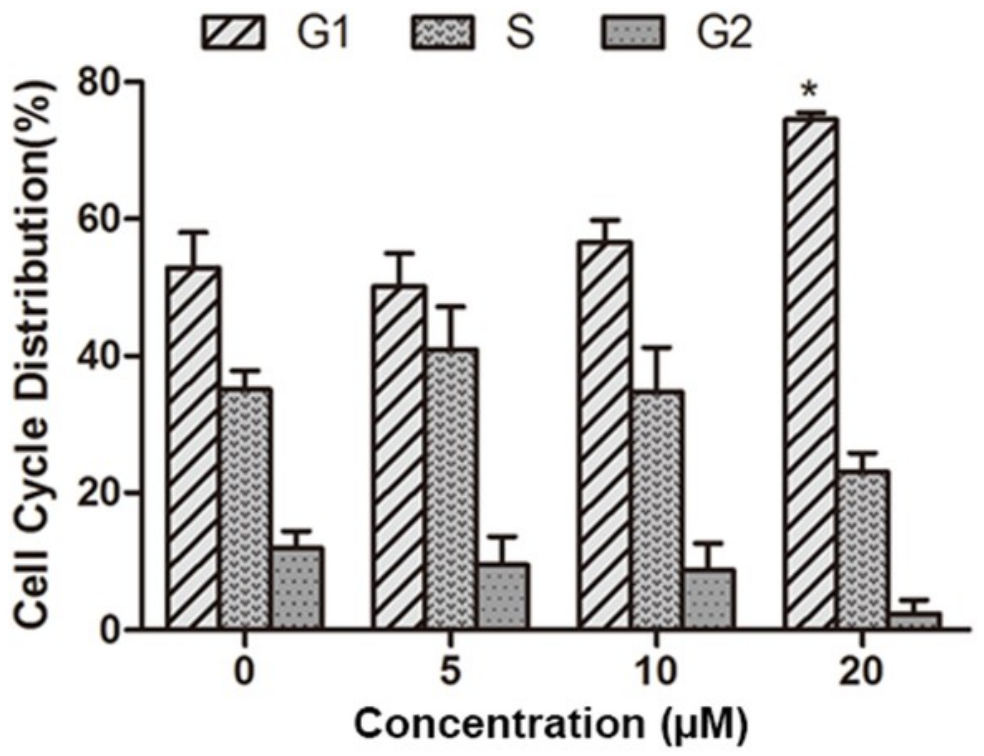




\section{Figure 3}

Fig. 3. Psoralidin showed little effect on apoptosis in A549 cells.

(A) Fluorescent staining of nuclei in A549 cells by Hoechst 33342. (B) Flow cytometry assay to detect apoptosis in A549 cells using Annexin V staining. There is no significant difference among control group and psoralidin treated groups. NS, no significant vs. Con. (C) The expression of apoptosis related proteins were analyzed by Western blot in A549 cells receiving psoralidin treatment. (D) The cell viability was measured by MTT assay after treatment with psoralidin in the absence or presence of Z-VAD-FMK or Ac-DEVD-CHO. ${ }^{* *} P<0.01$ vs. Con; NS, no significant vs. psoralidin alone-treated group. Con, control $<$ ?xml:namespace prefix = o ns = "urn:schemasmicrosoft-com:office:office" />
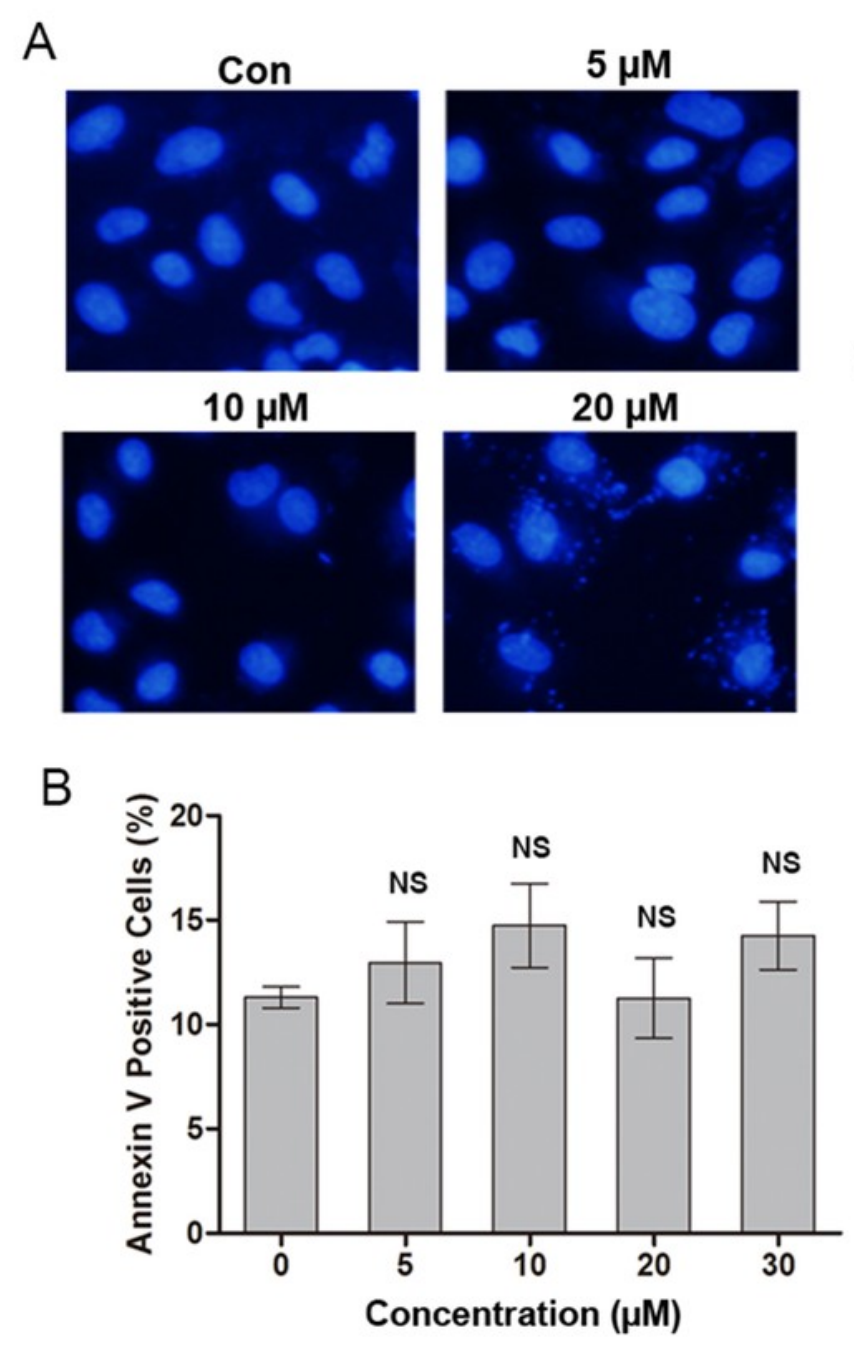

C
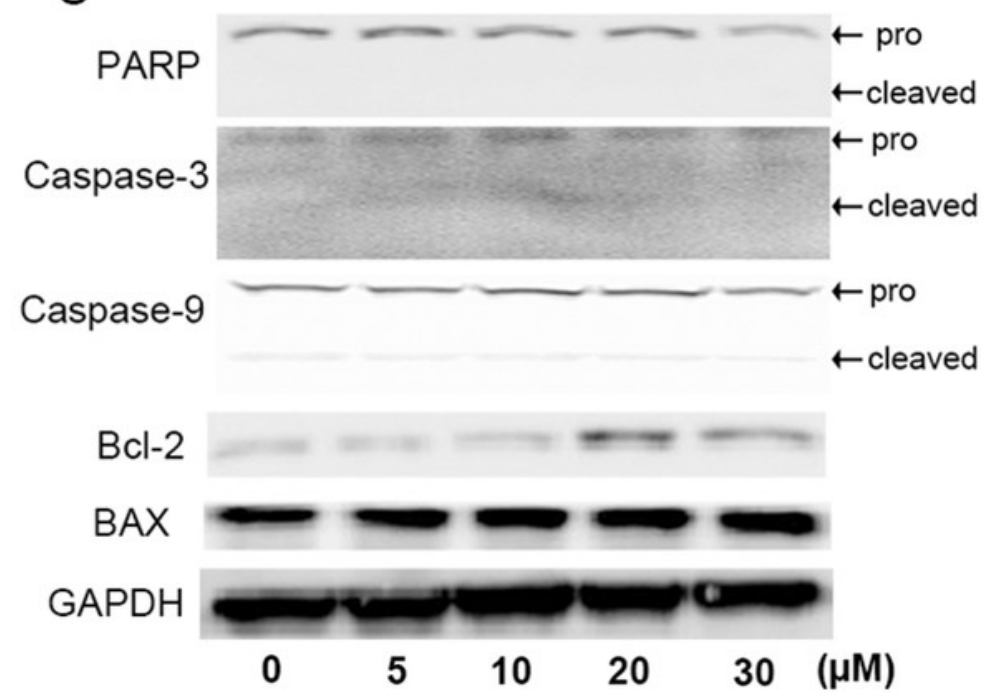

D

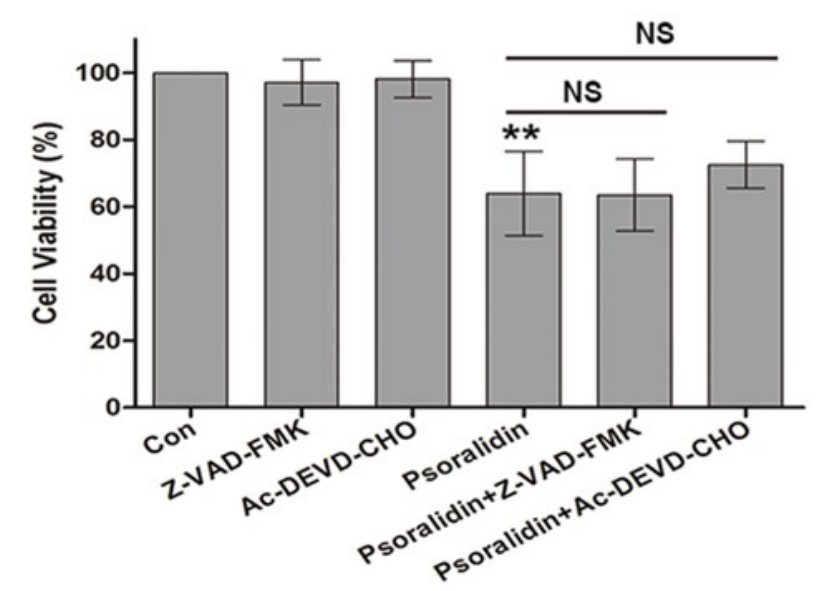




\section{Figure 4}

Fig. 4. Psoralidin induced autophagic cell death in A549 cells.

(A) Cells were stained with MDC after psoralidin treatment. (B) Immunofluerescence analysis of endogenous LC3 in psoralidin-treated A549 cells. (C) Cells were treated with indicated concentrations of psoralidin for $24 \mathrm{~h}$. The expression of LC3 was determined by Western blot. (D) Cells were treated with $20 \mu \mathrm{M}$ psoralidin for $6,12,24$ or $48 \mathrm{~h}$, expression of LC3 was determined by Western blot. (E) Cells were treated with psoralidin, 3-MA or a combination of both, expression of LC3 was analyzed by Western blot. (F) The cell viability was measured by MTT assay after treatment with psoralidin in the absence or presence of 3-MA. ${ }^{* \star} P<0.01 \mathrm{vs}$. Con, \#P<0.05 vs. psoralidin alone-treated group. Con, control $<$ ?xml:namespace prefix $=0 \mathrm{~ns}=$ "urn:schemasmicrosoft-com:office:office" /> 

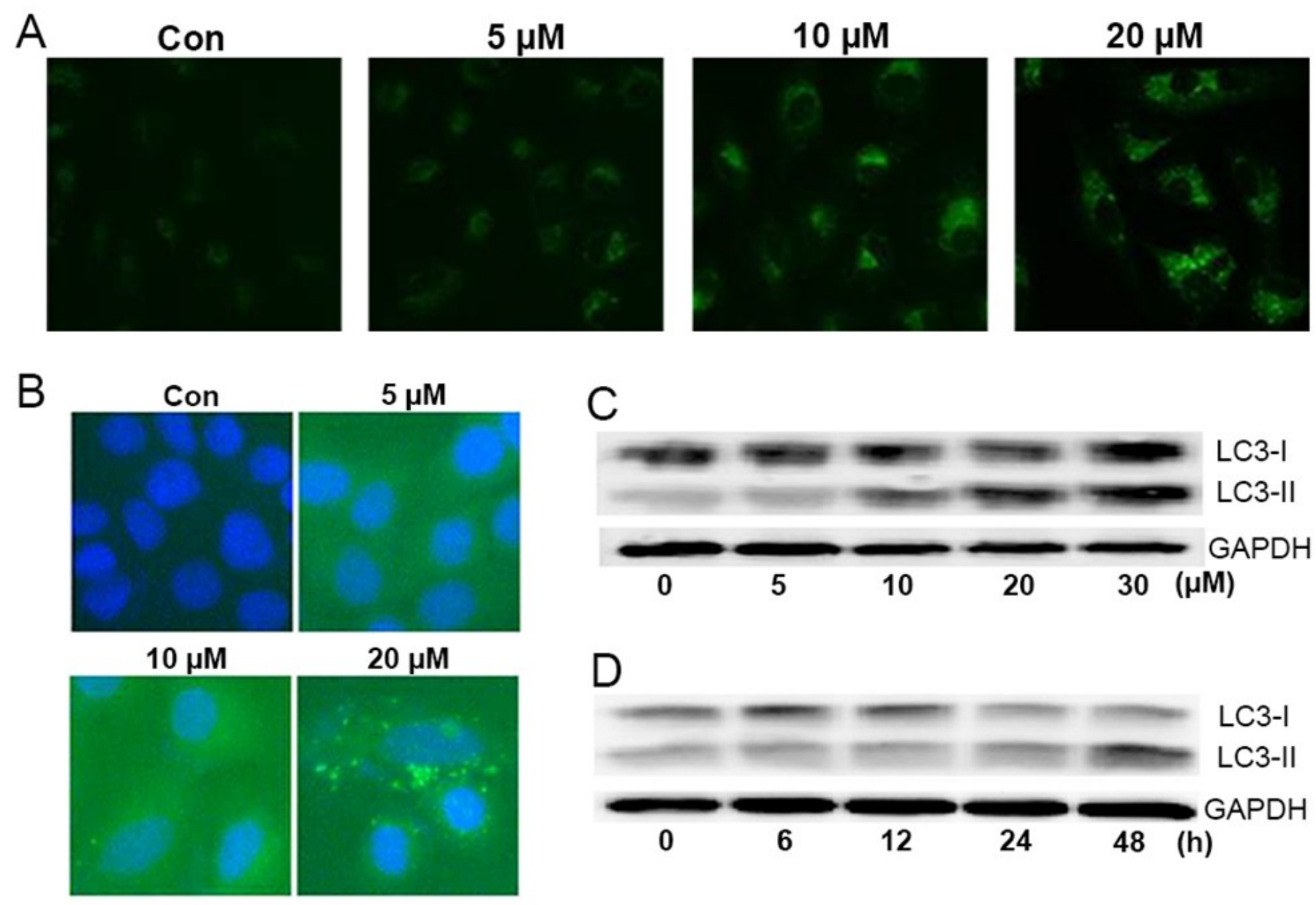

D

E

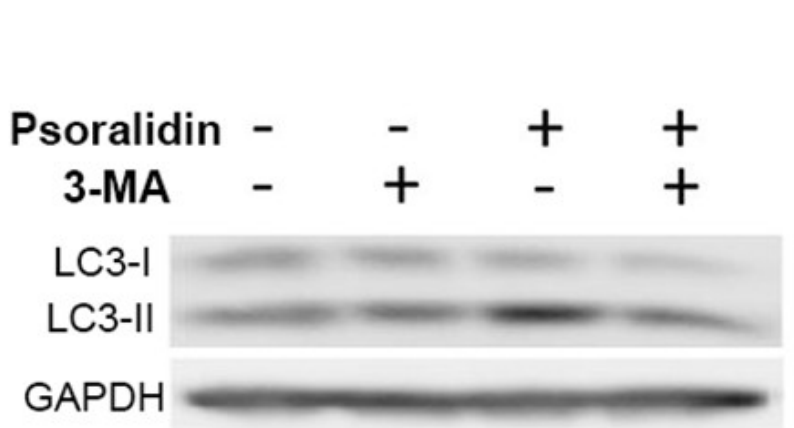

F

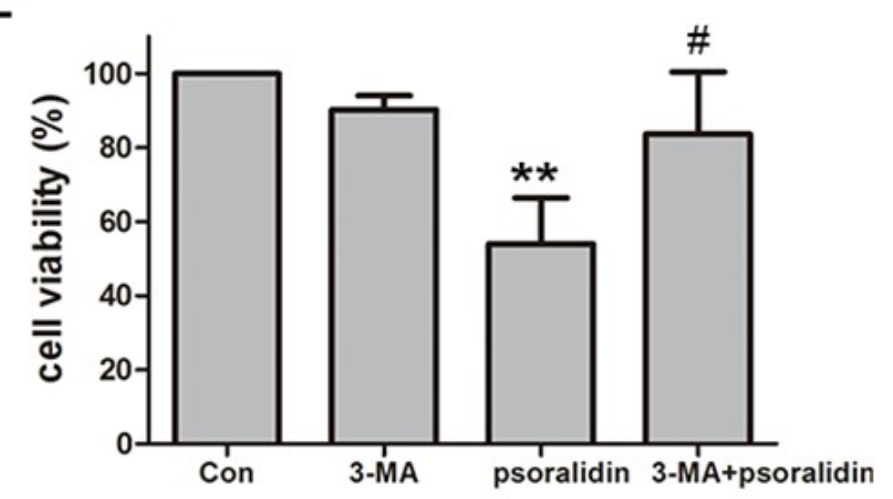




\section{Figure 5}

Fig. 5. Psoralidin induced ROS generation and NAC reversed psoralidin-induced autophagy and cell death.

(A) Cells were treated with indicated concentrations of psoralidin, and the intracellular ROS generation was detected as described in Materials and methods. ${ }^{*} P<0.05$ vs. Con and ${ }^{* \star} P<0.01$ vs. Con. (B) Cells were treated with $20 \mu \mathrm{M}$ psoralidin in the absence or presence of NAC. The intracellular ROS generation was detected, and data represent the means \pm SD from three independent experiments. ${ }^{\star *} P<0.01$ vs. Con, ${ }^{\#} P<0.05$ vs. psoralidin alone-treated group. (C) Cells were treated with $20 \mu \mathrm{M}$ psoralidin in the absence or presence of NAC. The expression of LC3 was analyzed by Western blot. (D) The cell viability was measured by MTT assay after psoralidin with treatment in the absence or presence of NAC. ${ }^{* *} P<0.01$ vs. Con, ${ }^{\#} P<0.05$ vs. psoralidin alone-treated group. Con, control $<$ ?xml:namespace prefix $=0 \mathrm{~ns}=$ "urn:schemasmicrosoft-com:office:office" />

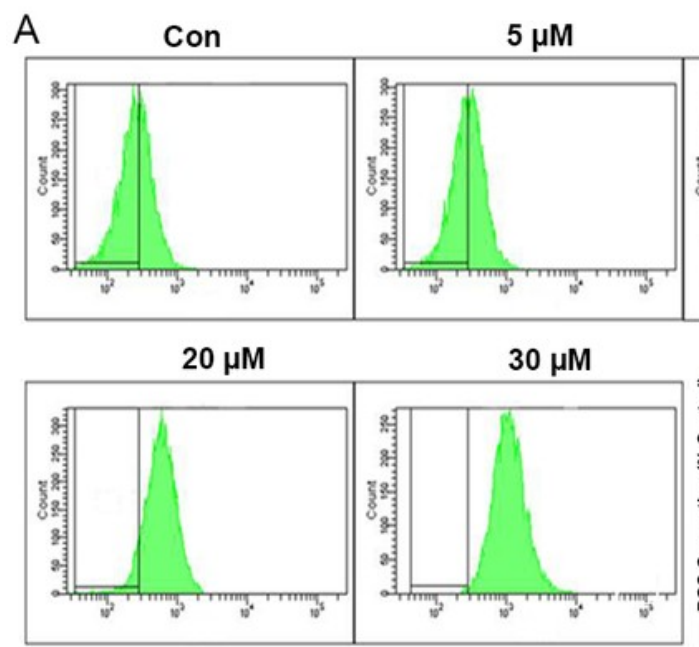

C
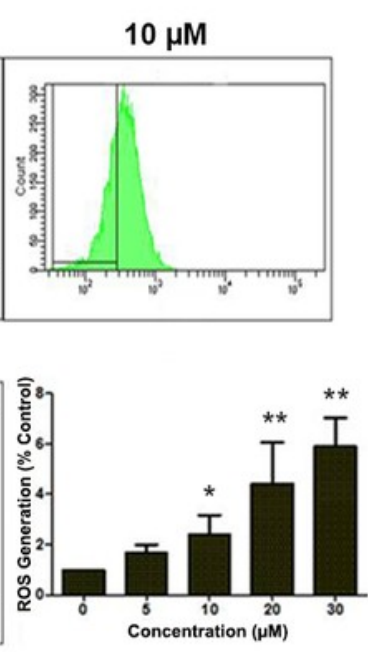

B
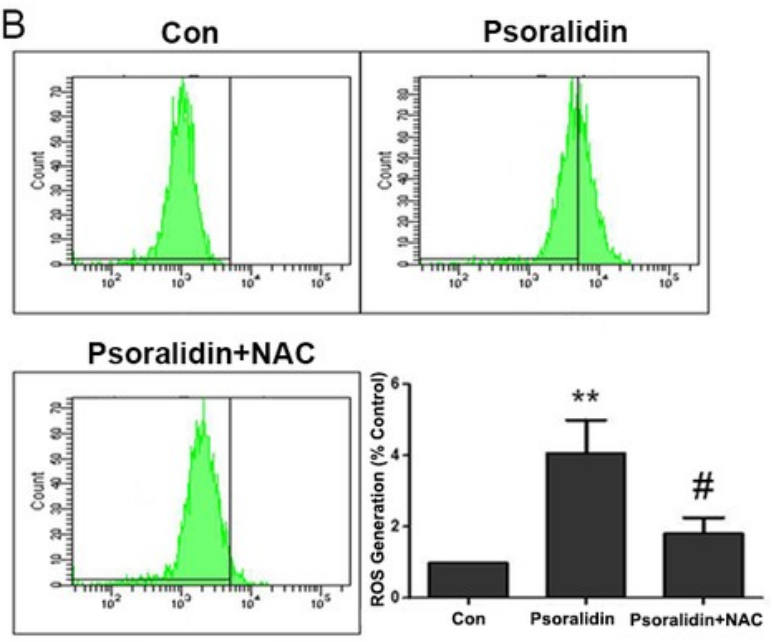

$\mathrm{D}$

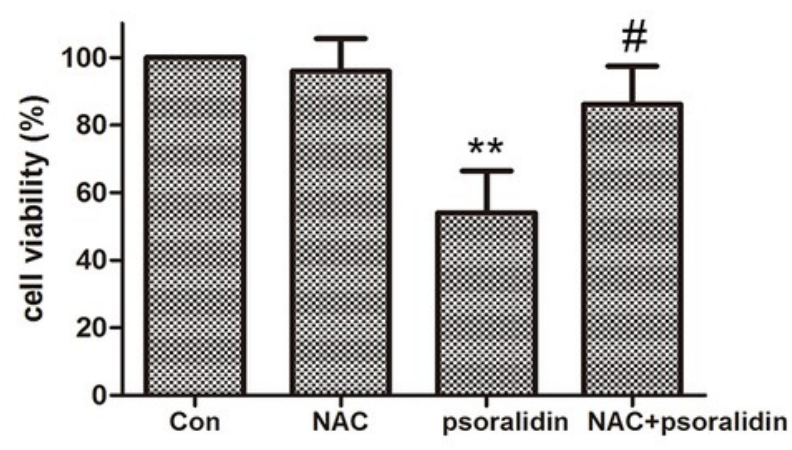




\section{Figure 6}

Fig. 6. Schematic diagram illustrates the underlying mechanism of psoralidin-induced cell death in A549 cells. $<$ ?xml:namespace prefix = o ns = "urn:schemas-microsoft-com:office:office" / >

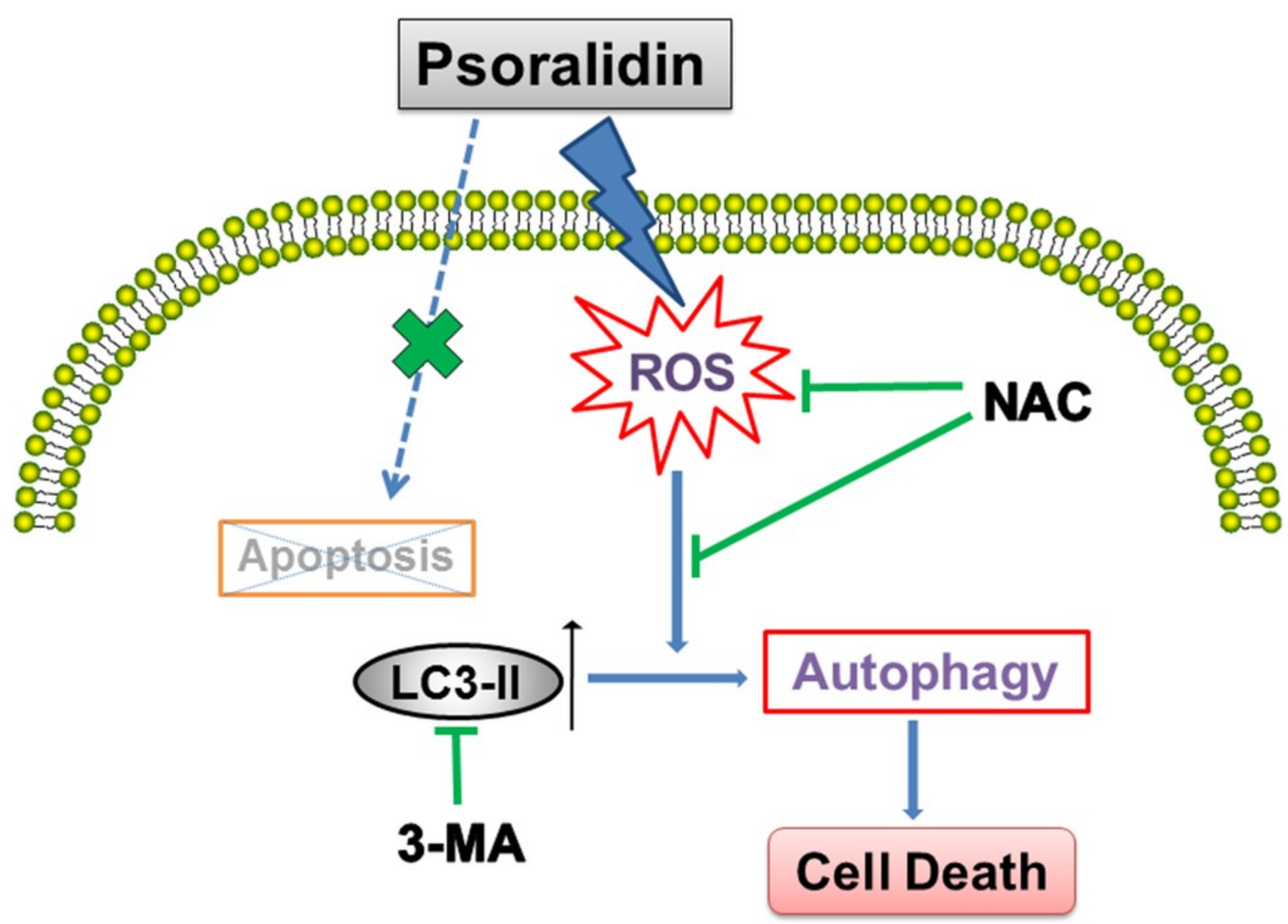

